\title{
Clinical Assessment of Re-Epithelialization using Methylene Blue Assay after Depigmentation under Surgical Microscope: A Case Report
}

\author{
Kriti Mehrotra Vijay ${ }^{1}$, Tanya Anil Jaitly ${ }^{2 *}$, Triveni MG $^{3}$, Tarun Kumar AB ${ }^{3}$, Dhoom Singh Mehta \\ ${ }^{1}$ Private Practitioner, Kota, Rajasthan, India. \\ 2*Postgraduate, Department of periodontics, Bapuji Dental College and Hospital, Davangere, India. \\ ${ }^{3}$ Professor, Department of periodontics, Bapuji Dental College and Hospital, Davangere, India. \\ ${ }^{4}$ Professor, Head of Department, Bapuji Dental College and Hospital, Davangere, India. \\ drtanyaaj@gmail.com
}

*Corresponding Author: Dr. Tanya Anil Jaitly, Room no 5, Department of Periodontics, Bapuji Dental College and Hospital, Davangere 557004, Karnataka, India.

\section{Abstract}

Aesthetic medicine has been an area of significant interest since ages, which is slowly but steadily garnering popularity owing to increased patient awareness.

The normal color of gingiva is coral pink with oral pigmentation being common among some races and ethnic groups. Melanin is one of the prime pigment responsible for gingival pigmentation and is of great aesthetic concern, especially in patients with high smile line. Periodontal plastic surgeries aim at improving the smile, with depigmentation being one of them. In this case report, evaluation of depigmentation using scalpel surgery and laser technique was done by clinical assessment of re-epithelialization. In this case, a 20year old

girl was treated for depigmentation using split mouth surgical protocol comparing scalpel surgery and laser technique.

Depigmentation using scalpel was done on the right side of the maxillary arch and Laser ablation on the left side. Post-operatively vitamin E oil was used as an adjunct to promote uneventful healing.

Patient was recalled regularly for the first week and the rate of epithelialization was assessed at chairside using methylene blue. Although healing with laser assisted depigmentation was initially slow but enhanced healing was appreciated. However, on the 7th day both the sites exhibited equivalent outcome. vitamin E could be a contributory factor leading to faster and enhanced healing and can be used as an adjunct to surgical procedures to escalate the healing process.

Keywords: Hyperpigmentation, visual perception, vitamin E oil, methylene blue, re-epithelialization, Lasers

\section{INTRODUCTION}

Smile is a vital component of a beautiful face and is responsible for $60-70 \%$ of its visual perception. A harmonious smile does not just come from beautiful lips but also a perfectly healthy gingival frame and well aligned, healthy, natural teeth. ${ }^{1}$

The color of healthy gingiva varies from pink to bluish purple ${ }^{2}$ and between these normallimits is a spectrum of colors depending on the intensity of melanogenesis, degree of keratinization and vascularity. Melanin, carotene, reduced hemoglobin and oxy-hemoglobin are the prime pigments contributing to the normal color of the oral mucosa.

Excessive deposition of melanin located in the basal and supra-basal cell layers of the epithelium causes gingival hyperpigmentation. The occurrence and distribution pattern of gingival hyper pigmentation has been diverse showing inter and intragroup variability within ethnic groups which is generally correlated with cutaneous pigmentation but not confined 
Clinical Assessment of Re-Epithelialization using Methyleneblue Assay after Depigmentation under Surgical Microscope: A Case Report

to heavily pigmented races. ${ }^{3}$ Although physiologic and ethnic melanin hyperpigmentation is not a pathology but "black gums" may lead to aesthetic complaints, particularly in patients with a high smile line. ${ }^{4}$

Perio-aesthetic treatment modalities strive to achieve a harmonious inter-relationship of the pink with white, with gingival depigmentation being one of them. Various treatment alternatives are available such as diamond bur surgery, scalpel technique, electrosurgery, cryosurgery, Lasers and soft tissue augmentation. ${ }^{5}$ The foremost indication for depigmentation is patient's demand for improved aesthetics. The selection of technique is usually based on clinical experience, availability of armamentarium and individual preferences.

This case report demonstrates Split mouth evaluation of depigmentation using scalpel surgery and Laser technique based on assessment of clinical healing through methylene blue dye. In addition, efficacy of topical vitamin $\mathrm{E}$ oil as an adjunct to healing and rate of re epithelialization was assessed.

\section{Materials AND Methods}

A 20year old girl, reported to the Department of Periodontics, with a chief complaint of black gums visible on smiling. Region to be depigmented was evaluated as Class 3 DOPI (Dummet's Oral Pigmentation Index) Assessment Index ${ }^{6}$ (Fig 1).

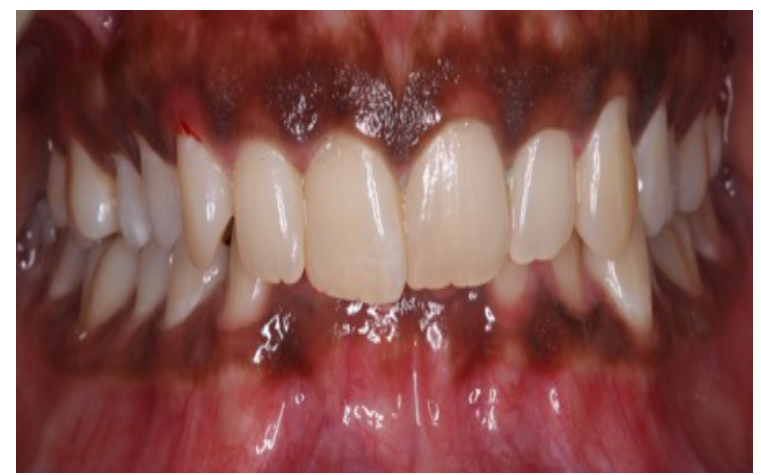

Figure1. Pre-operative: labial view of pigmented gingiva as Class 3 DOPI Assessment Index

Medical and personal history were non-contributory and patient was informed about the treatment after which consent was taken. Blood investigations were within normal limits.

On the day of procedure, random allocation of surgical depigmentation was done on the right side and laser assisted depigmentation on the left side of the maxillary arch using surgical microscope. Local anesthesia (Lignocaine 1:200000) was administered and gingival de-epithelization was performed using micro blades \#63 and \#64 in a scraping motion (Fig 2) with concomitant saline irrigation.

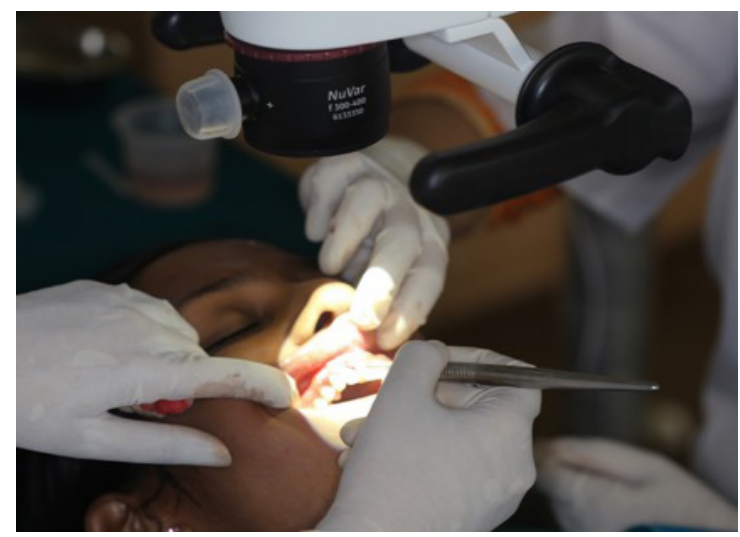

Figure 2. Surgical ablation done under surgical micro scope from \# 8 to \# 5

On the left side of the maxillary arch, a diode laser with beam diameter of $320 \mu \mathrm{m}, 810 \mathrm{~nm}$ wavelength was operated at 1.4 watt in a pulsed mode (Fig 3) under topical anesthesia. Laser ablation was started from the midline in a linear motion using the 'brush technique ${ }^{7}$ (Fig 4). Charred tissue was removed using sterile nonwoven gauze soaked in saline. After completion of the procedure vitamin E oil was applied on both treated sites (Fig 6) and instructed to apply Vitamin E twice a day for a week.

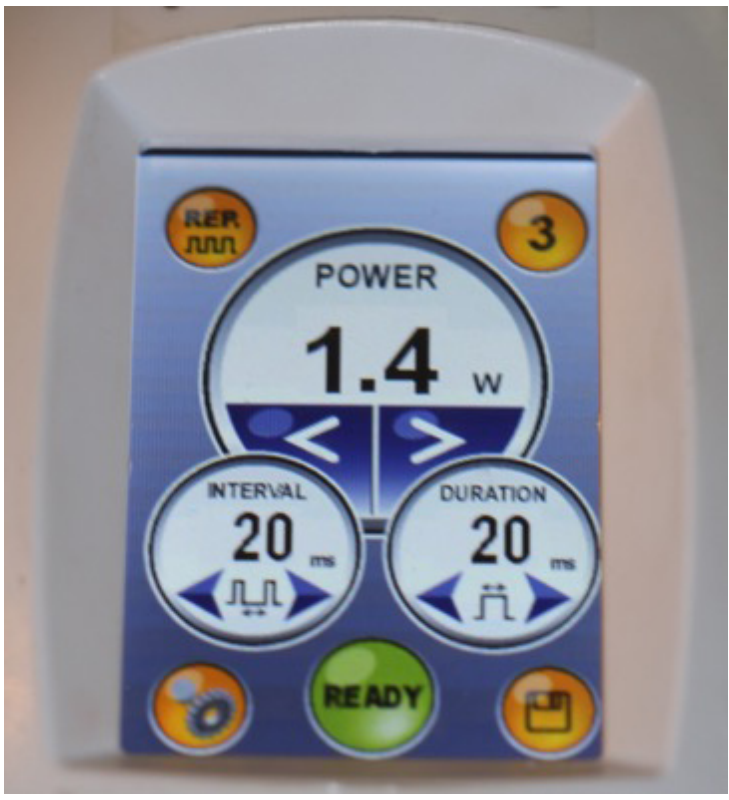

Figure 3. Laser parameters 
Clinical Assessment of Re-Epithelialization using Methyleneblue Assay after Depigmentation under Surgical Microscope: A Case Report

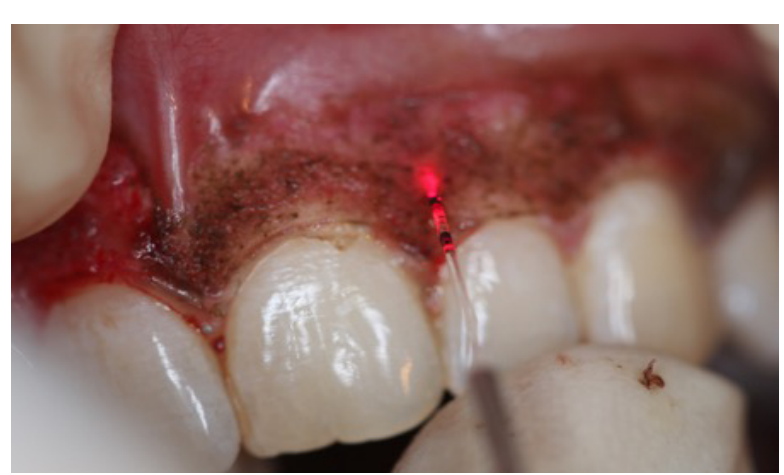

Figure 4. Laser assisted gingival depigmentation done from \# 9 to \# 12

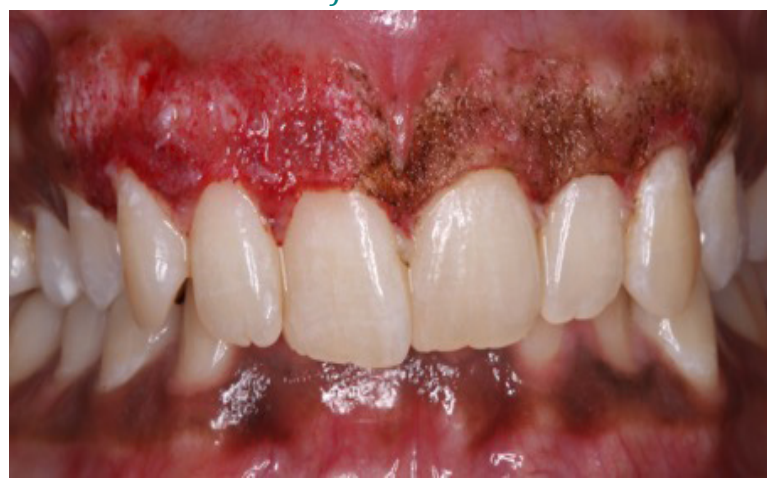

Figure 5. Post-operative view (Right: scalpel technique ; Left: laser assisted)

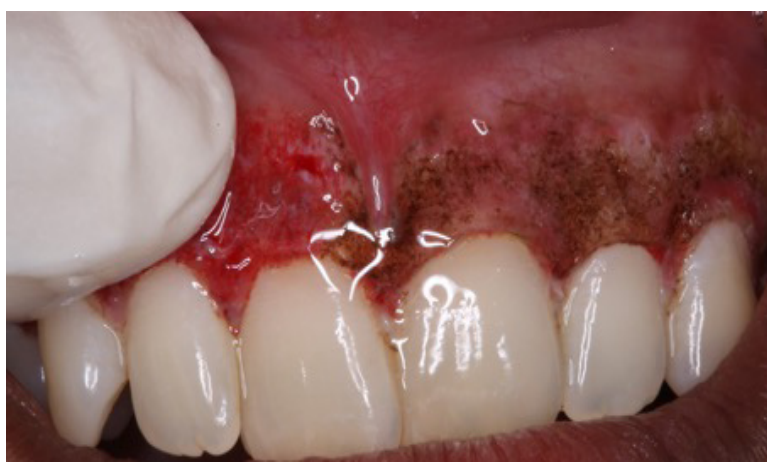

Figure 6. Topical Vitamin E oil application

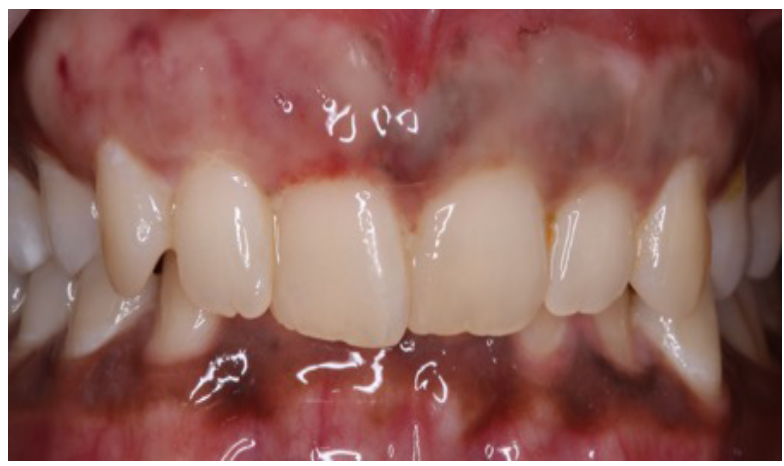

Figure 7. Day 1 post-operative labial view
Patient was evaluated daily for a week and the rate of epithelialization was assessed using methylene blue (Fig 8-13). Patient was asked to rinse with the dye and immediately the stained area was swabbed gently with a damp gauze which removed the dye from the surface of normal epithelium, whereas nonviable tissue and granulation tissue remain stained.

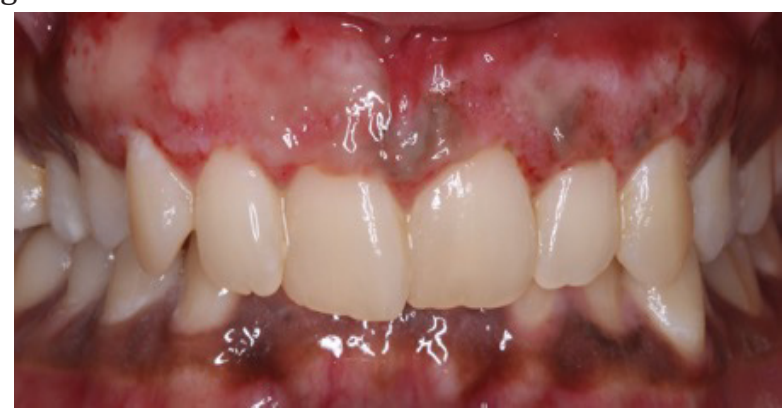

Figure 8. Day 2 post-operative labial view

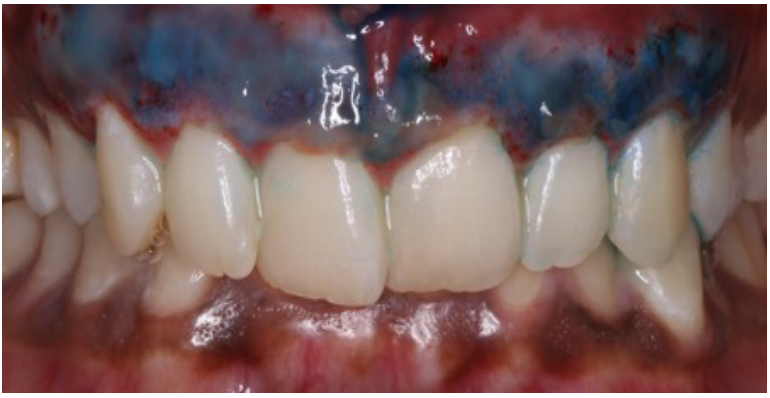

Figure 9. Healing checked on 2nd day. Note the increased uptake of dye by the damaged tissue

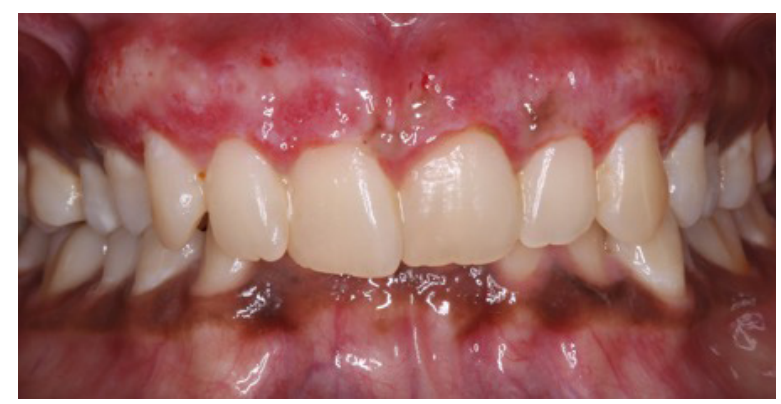

Figure 10. Day 4 post-operative labial view

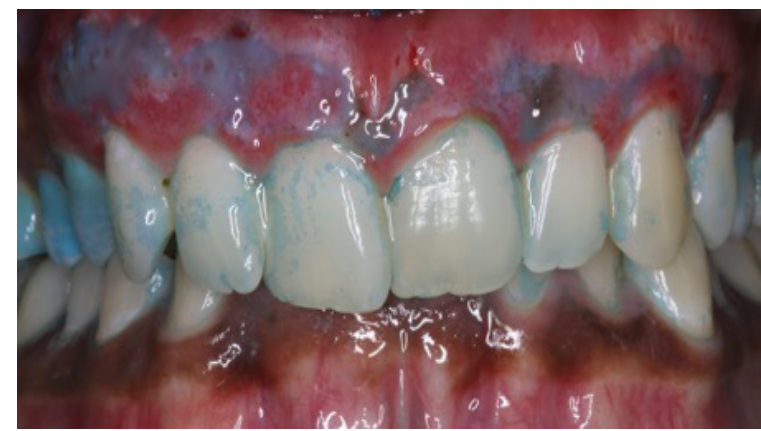

Figure 11. Healing checked on 4th day. Note the gradual decrease in uptake of the dye by the healing tissue 


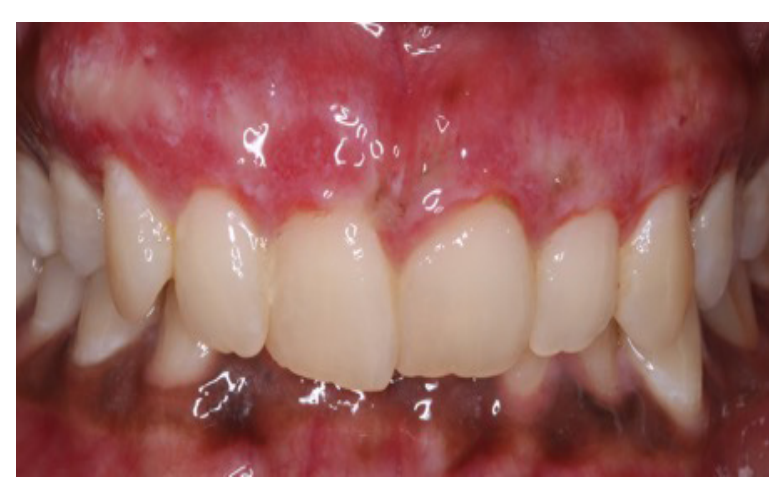

Figure 12. Day 5 post-operative labial view

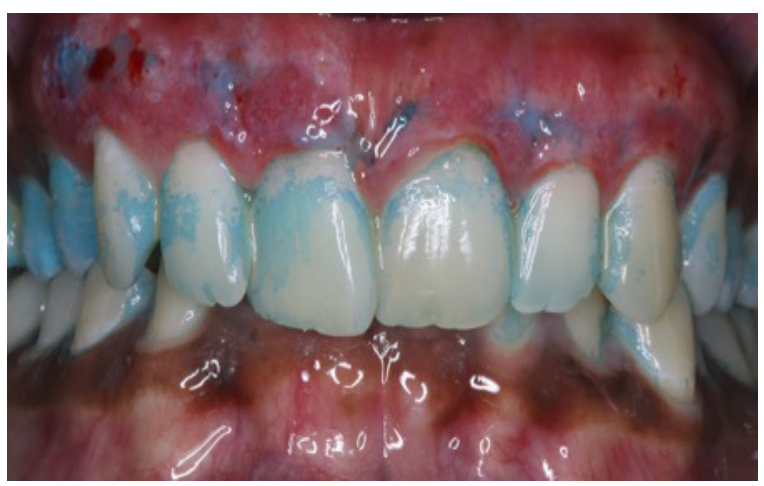

Figure 13. Healing checked on the 5th day

\section{RESULTS}

The uptake of methylene blue dye by the tissue gradually decreased indicating complete reepithelialization within 1 week (Fig 15).

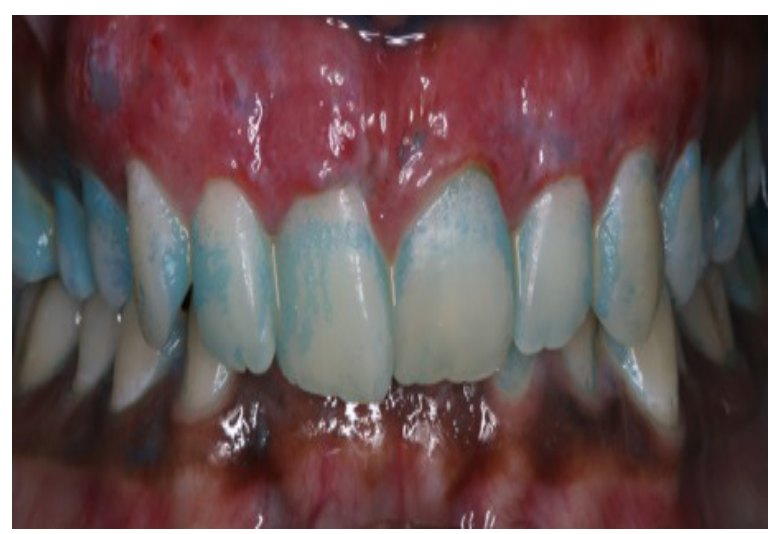

Figure 14. Healing checked on the 6th day showing little or no uptake of the dye by tissues thus indicating re-epithelialization

Though the rate of epithelization assessed through chairside use of methylene blue dye showed initially slower rate with laser assisted depigmentation as compared to surgical method, on the 7th day both the sites exhibited equivalent outcome (Fig 15)

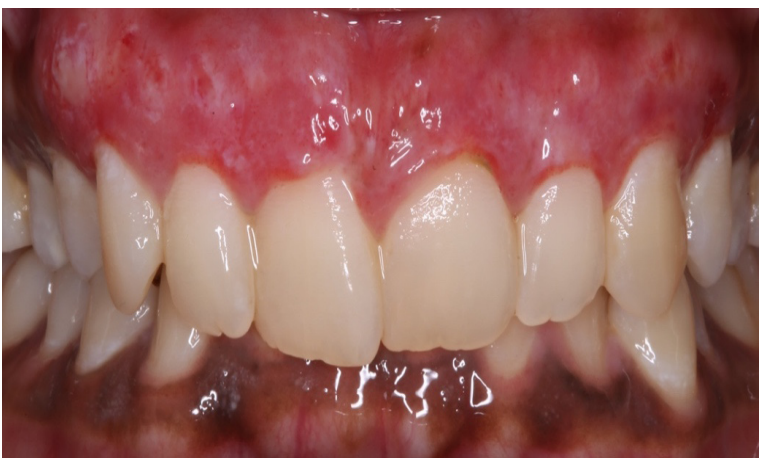

Figure 15. Day 7 post-operative view

\section{Discussion}

Melanin buildout is regulated by the individual's genetic makeup. Melaninissynthesizedbymelanocytes which are dendritic cells of neuro-ectodermal origin by converting tyrosine to melanoprotein (melanin) which is transferred to keratinocytes via melanosomes. It is deposited in the basal layer of the oral epithelium. ${ }^{8}$

The average thickness of epithelium is $0.31 \pm 0.05 \mathrm{~mm}^{9}$, thus for complete depigmentation it is imperative to remove tissue more than $0.31 \mathrm{~mm}$, i.e. the epithelium and a part of connective tissue which will subsequently heal by secondary intention. The new epithelium formed is devoid of melanin pigmentation. ${ }^{10}$

The use of surgical microscope in this case helped focus the area of concern that allowed solitary units of pigmentation to be appreciated. It delivers high intensity, cool and color correct light that makes the operator comfortable thus increasing the efficiency.

According to Lin et $\mathrm{al}^{11}$ in a systematic review on treatment modalities of gingival depigmentation, Laser offers optimal predictability in depigmentation following cryosurgery and electrosurgery. Diode lasers $(810 \mathrm{~nm})$ have the highest clinical effect on melanin as its wavelength allows high levels of energy to be absorbed by soft tissue, water and chromophores like melanin and oxyhemoglobin. Here, superior healing was appreciated on both the sides on the 7th day with initial delay in wound healing on the laser treated site which could be attributed to the fact that laser beam causes thermal coagulation that seals blood vessels and lymphatic channels less than $1 \mathrm{~mm}$ in diameter resulting in delayed proliferation of capillaries. ${ }^{12}$ These findings are similar to the study conducted by Suragimath et. $\mathrm{al}^{13}$ wherein wound healing did 
Clinical Assessment of Re-Epithelialization using Methyleneblue Assay after Depigmentation under Surgical Microscope: A Case Report

not show statistical difference between the two techniques.

During re-epithelialization, fibroblasts migrate from the wound edge into the wound bed at a rate of 12 $21 \mu \mathrm{m} / \mathrm{hr}$. As keratinocytes migrate more easily over a moist wound surface, this is significantly impacted by moisture-rich environment which is provided by Vitamin E oil. This work was supported by Zampieri and colleagues. ${ }^{14}$

Vitamin E is also a potent lipid-soluble antioxidant, application of which is effective as both laser and surgical treatments increase the production of free radicals which are exceedingly reactive and harmful molecules. It also interferes with lipid peroxidation of melanocyte membranes and increases the intracellular glutathione content, and inhibition of tyrosinase could explain its depigmenting effect ${ }^{15}$. Thus, regular topical application of vitamin E might be a contributing factor resulting in faster and better healing.

Methylene blue is a vital dye that has been used to assess the progress of wound healing ${ }^{16}$. It has also been used effectively as an early diagnostic marker for oral precancerous and cancerous lesions. ${ }^{17,18}$ It stains the negatively charged molecules, including the DNA, within damaged cells but does not stain the nonabsorptive epithelia. This makes it a simple yet effective technique to track course of re-epithelialization ${ }^{19}$. As seen in the present case, methylene blue dye uptake gradually decreased over a period of 1 week indicating re-epithelialization.

\section{CONCLUSION}

Chairside assessment of re-epithelialization using methylene blue after a surgery can become a part of day to day practice that helps surgeon evaluate the rate and extent of healing owing to its low toxicity and it being a simple, inexpensive and rapid technique. Exact determination of re-epithelialization and its correlation to methylene blue loss requires more sophisticated methods.

vitamin E oil can be used as an adjunct to enhance healing but further research with a large sample size using control is required to substantiate its role in wound healing.

Thus, modern dentistry aims to achieve the best possible result with minimal tissue invasion, thus giving the patient a beautiful smile.

\section{REFERENCES}

[1] André P. Saadoun. Esthetic Soft Tissue Management of Teeth and Implants: John Wiley \& Sons, Ltd; 2013:1.

[2] Dummett CO, Barens G. Pigmentation of the oral tissues: a review of the literature. J Periodontol. 1967 Sep-Oct;38(5):369-78. Review.

[3] Dummett Co. Oral Pigmentation. FirstSymposium on oral pigmentation. J Periodontol 1960; 31: 356-360.

[4] Zarone F, Leone R, Ferrari M, Sorrentino R. Treatment Concept for a Patient with a High Smile Line and Gingival Pigmentation: A Case Report. Int J Periodontics Restorative Dent. 2017 Mar/Apr;37(2):e142-e148.

[5] Wise RJ, Chen CY, Kim DM. Treatment of Physiologic Gingival Pigmentation with Surgical Blade: A 25-Year Follow-up. Int J Periodontics Restorative Dent. 2018;38(Suppl):s45-s48.

[6] DumettCo,Gupta OP.Estimating the epidemiology of oral pigmentation. J Natl Med Assoc. 1964; 56: 419-20.

[7] Tal H, Oegiesser D, Tal M. Gingival depigmentation by erbium: YAG laser: clinical observations and patient responses. J Periodontol. 2003; 74 (11) :1660-7.

[8] Bergamaschi 0, Kon S, Doine AI, Ruben MP. Melanin repigmentation after gingivectomy: a 5-year clinical and transmission electron microscopic study in humans. Int J Periodontics Restorative Dent. 1993;13(1):85-92.

[9] Schroeder HE. Differentiation of Human Oral Stratified Epithelia. Basel: Karger; 1981.

[10] Gupta G, Kumar A, Khatri M, Puri K, Jain D, Bansal M. Comparison of two different depigmentation techniques for treatment of hyperpigmented gingiva. J Indian Soc Periodontol. 2014 Nov-Dec ;18(6):705-9.

[11] Lin YH, Tu YK, Lu CT, Chung WC, Huang CF, Huang MS, Lu HK. Systematic review of treatment modalities for gingival depigmentation: a random 
Clinical Assessment of Re-Epithelialization using Methyleneblue Assay after Depigmentation under Surgical Microscope: A Case Report

effects poisson regression analysis. J Esthet Restor Dent. 2014 May-Jun;26(3):162-78

[12] Luomanen M. A comparative study of healing of laser and scalpel incision wounds in rat oral mucosa. Scand J Dent Res. 1987;95(1):65-73. (12)

[13] Suragimath G, Lohana MH, Varma S. A Split Mouth Randomized Clinical Comparative Study to Evaluate the Efficacy of Gingival Depigmentation Procedure Using Conventional Scalpel Technique or Diode Laser. J Lasers Med Sci. 2016 Fall;7(4):227-232.

[14] Zampieri N, Zuin V, Burro R, Ottolenghi A, Camoglio FS. A prospective study in children: Pre- and post-surgery use of vitamin E in surgical incisions. J Plast Reconstr Aesthet Surg. 2010;63(9):1474-8.

[15] Mohammad Abid Keen, Iffat Hassan. Indian Dermatol Online. 2016; 7(4): 311-315.
[16] Dorafshar AH, Gitman M, Henry G, Agarwal S, Gottlieb LJ. Guided surgical debridement: staining tissues with methylene blue. J Burn Care Res. 2010;31(5):791-4.

[17] Chen YW, Lin JS, Wu CH, Lui MT, Kao SY, Fong Y. Application of in vivo stain of methylene blue as a diagnostic aid in the early detection and screening of oral squamous cell carcinoma and precancer lesions. J Chin Med Assoc. 2007 Nov;70(11):497-503.

[18] Lejoy A, Arpita R, Krishna B, Venkatesh N. Methylene Blue as a Diagnostic Aid in the Early Detection of Potentially Malignant and Malignant Lesions of Oral Mucosa. Ethiop J Health Sci. 2016 May;26(3):201-8.

[19] Milyavsky M, Dickie R. Methylene Blue Assay for Estimation of Regenerative Re Epithelialization In Vivo. Microsc Microanal. 2017;23(1):113-121.

Citation: Kriti Mehrotra Vijay, Tanya Anil Jaitly, Triveni MG, Tarun Kumar AB, Dhoom Singh Mehta. Clinical Assessment of Re-Epithelialization using Methyleneblue Assay after Depigmentation under Surgical Microscope: A Case Report. Archives of Dentistry and Oral Health. 2018; 1(2): 26-31.

Copyright: (c) 2018 Kriti Mehrotra Vijay, Tanya Anil Jaitly, Triveni MG, Tarun Kumar AB, Dhoom Singh Mehta. This is an open access article distributed under the Creative Commons Attribution License, which permits unrestricted use, distribution, and reproduction in any medium, provided the original work is properly cited. 